EUROPEAN ORGANIZATION FOR NUCLEAR RESEARCH

CERN-PPE/95-31

September 29, 1995

hep-ex/9509014

\title{
LARGE ENHANCEMENT OF DEUTERON POLARIZATION WITH FREQUENCY MODULATED MICROWAVES
}

The Spin Muon Collaboration (SMC)

\begin{abstract}
We report a large enhancement of 1.7 in deuteron polarization up to values of 0.6 due to frequency modulation of the polarizing microwaves in a two liters polarized target using the method of dynamic nuclear polarization. This target was used during a deep inelastic polarized muon-deuteron scattering experiment at CERN. Measurements of the electron paramagnetic resonance absorption spectra show that frequency modulation gives rise to additional microwave absorption in the spectral wings. Although these results are not understood theoretically, they may provide a useful testing ground for the deeper understanding of dynamic nuclear polarization.
\end{abstract}


B. Adeva ${ }^{22}$, E. Arik ${ }^{3}$ S. Ahmad ${ }^{20}$, A. Arvidson ${ }^{25}$, B. Badelek ${ }^{25,27}$, M.K. Ballintijn ${ }^{17}$,

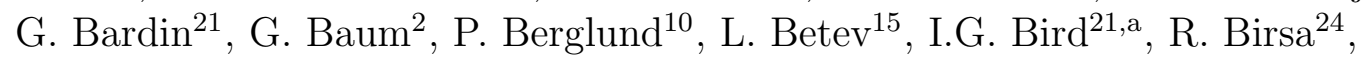
P. Björkholm ${ }^{25}$, B.E. Bonner ${ }^{20}$, N. de Botton ${ }^{21}$, M. Boutemeur ${ }^{28, b}$, F. Bradamante ${ }^{24}$, A. Bressan ${ }^{24}$, A. Brüll ${ }^{8, c}$, J. Buchanan ${ }^{20}$, S. Bültmann ${ }^{2}$, E. Burtin ${ }^{21}$, C. Cavata ${ }^{21}$, J.P. Chen ${ }^{26}$, J. Clement ${ }^{20}$, M. Clocchiatti ${ }^{24}$, M.D. Corcoran ${ }^{20}$, D. Crabb ${ }^{26}$,

J. Cranshaw ${ }^{20}$, T. Çuhadar ${ }^{3}$, S. Dalla Torre ${ }^{24}$ A. Deshpande ${ }^{28}$, R. van Dantzig ${ }^{17}$, D. Day ${ }^{26}$, S. Dhawan ${ }^{28}$, C. Dulya ${ }^{5}$, A. Dyring ${ }^{25}$, S. Eichblatt ${ }^{20}$, J.C. Faivre ${ }^{21}$, D. Fasching ${ }^{19}$, F. Feinstein ${ }^{21}$, C. Fernandez ${ }^{22,11}$, B. Frois ${ }^{21}$, C. Garabatos ${ }^{22}$, J.A. Garzon ${ }^{2,11}$, T. Gaussiran ${ }^{20}$, M. Giorgi ${ }^{24}$, E. von Goeler ${ }^{18}$, I.A. Goloutvin ${ }^{12}$, A. Gomez ${ }^{22,11}$, G. Gracia ${ }^{22}$, N. de Groot ${ }^{17}$, M. Grosse Perdekamp ${ }^{5}$, E. Gülmez ${ }^{3}$ D. von Harrach ${ }^{13}$, T. Hasegawa ${ }^{16, \mathrm{~d}}$, P. Hautle ${ }^{7, \mathrm{e}}, \mathrm{N} . \mathrm{Hayashi}^{16}$, C.A. Heusch ${ }^{6}$, N. Horikawa ${ }^{16}$, V.W. Hughes ${ }^{28}$, G. Igo ${ }^{5}$, S. Ishimoto ${ }^{16, f}$, T. Iwata ${ }^{16}$, M. de Jong ${ }^{7}$, E.M. Kabuß ${ }^{13}$, T. Kageya ${ }^{16}$, R. Kaiser ${ }^{8}$, A. Karev ${ }^{12}$, H.J. Kessler ${ }^{8}$, T.J. Ketel ${ }^{17}$, I. Kiryushin ${ }^{12}$, A. Kishi ${ }^{16}$, Yu. Kisselev ${ }^{12}$, L. Klostermann ${ }^{17}$, D. Krämer ${ }^{2}$, V. Krivokhijine ${ }^{12}$, V. Kukhtin ${ }^{12}$, J. Kyynäräinen ${ }^{7,10}$, M. Lamanna ${ }^{24}$, U. Landgraf ${ }^{8}$, K. Lau ${ }^{11}$, T. Layda ${ }^{6}$, J.M. Le Goff ${ }^{21}$, F. Lehar ${ }^{21}$, A. de Lesquen ${ }^{21}$, J. Lichtenstadt ${ }^{23}$, T. Lindqvist ${ }^{25}$, M. Litmaath ${ }^{17}$, S. Lopez-Ponte ${ }^{22,11}$, M. Lowe ${ }^{20}$, A. Magnon ${ }^{7,21}$, G.K. Mallot ${ }^{7,13}$, F. Marie ${ }^{21}$, A. Martin ${ }^{24}$, J. Martino ${ }^{21}$, T. Matsuda ${ }^{16}$, B. Mayes ${ }^{11}$, J.S. McCarthy ${ }^{26}$, K. Medved ${ }^{12}$ G. van Middelkoop ${ }^{17}$, D. Miller ${ }^{19}$, J. Mitchell ${ }^{26}$, K. Mori ${ }^{16}$, J. Moromisato ${ }^{18}$, G.S. Mutchler ${ }^{20}$, A. Nagaitsev ${ }^{12}$, J. Nassalski ${ }^{27}$, L. Naumann ${ }^{7}$, B. Neganov ${ }^{12}$, T.O. Niinikoski ${ }^{7}$, J.E.J. Oberski ${ }^{17}$, A. Ogawa ${ }^{18}$, S. Okumi ${ }^{16}$, C.S. Özben. ${ }^{4}$, A. Penzo ${ }^{24}$, C.A. Perez ${ }^{22}$, F. Perrot-Kunne ${ }^{21}$,

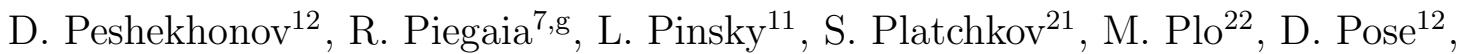
H. Postma ${ }^{17}$, J. Pretz ${ }^{13}$, T. Pussieux ${ }^{21}$, J. Pyrlik ${ }^{11}$, I. Reyhancan ${ }^{3}$, J.M. Rieubland ${ }^{7}$, A. Rijllart ${ }^{7}$, J.B. Roberts ${ }^{20}$, S.E. Rock ${ }^{1}$, M. Rodriguez ${ }^{22}$, E. Rondio ${ }^{27}$, O. Rondon ${ }^{26}$, L. Ropelewski ${ }^{27}$, A. Rosado ${ }^{15}$, I. Sabo ${ }^{23}$, J. Saborido ${ }^{22}$, G. Salvato ${ }^{24}$, A. Sandacz ${ }^{27}$, D. Sanders ${ }^{11}$, I. Savin ${ }^{12}$, P. Schiavon ${ }^{24}$, K.P. Schüler ${ }^{28, h}$, R. Segel ${ }^{19}$, R. Seitz ${ }^{13, q}$, Y. Semertzidis ${ }^{7}$, S.Sergeev ${ }^{12}$, F.Sever ${ }^{17}$, P. Shanahan ${ }^{19}$, E. Sichtermann ${ }^{17}$, G. Smirnov ${ }^{12}$, A. Staude ${ }^{15}$, A. Steinmetz ${ }^{13}$, H. Stuhrmann ${ }^{9}$, K.M. Teichert ${ }^{15}$, F. Tessarotto ${ }^{24}$,

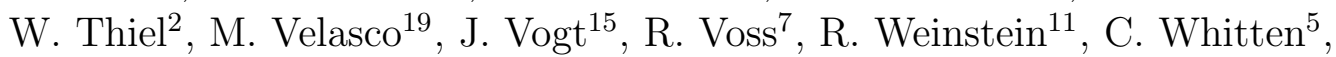
R. Willumeit ${ }^{9}$, R. Windmolders ${ }^{14}$, W. Wislicki ${ }^{27}$, A. Witzmann ${ }^{8}$, A. Yañez ${ }^{22}$, N.I. Zamiatin ${ }^{12}$, A.M. Zanetti ${ }^{24}$, J. Zhao ${ }^{9}$, 
1) The American University, Washington, D.C. 20016, USA

2) University of Bielefeld, Physics Department, 33615 Bielefeld, Germany ${ }^{\mathrm{i}}$

3) Bogazi ci University and Ceknece Nuclear Research Center, Istanbul, Turkey

4) Istanbul Technical University, Istanbul, Turkey

5) University of California, Department of Physics, Los Angeles, 90024 CA, USA ${ }^{j}$

6) University of California, Institute of Particle Physics, Santa Cruz, 95064 CA, USA

7) CERN, 1211 Geneva 23, Switzerland

8) University of Freiburg, Physics Department, 79104 Freiburg, Germany ${ }^{\mathrm{i}}$

9) GKSS, 21494 Geesthacht, Germany ${ }^{\mathrm{i}}$

10) Helsinki University of Technology, Low Temperature Laboratory, 02150 Espoo, Finland

11) University of Houston, Department of Physics, Houston, 77204-5504 TX, and Institute for Beam Particle Dynamics, Houston, 77204-5506 TX, USA ${ }^{\mathrm{j}, \mathrm{k}}$

12) JINR, Laboratory of Super High Energy Physics, Dubna, Russia

13) University of Mainz, Institute for Nuclear Physics, 55099 Mainz, Germany ${ }^{i}$

14) University of Mons, Faculty of Science, 7000 Mons, Belgium

15) University of Munich, Physics Department, 80799 Munich, Germany ${ }^{\mathrm{i}}$

16) Nagoya University, Department of Physics, Furo-Cho, Chikusa-Ku, 464 Nagoya, Japan ${ }^{1}$

17) NIKHEF, Delft University of Technology, FOM and Free University, 1009 AJ Amsterdam, The Netherlands ${ }^{\mathrm{m}}$

18) Northeastern University, Department of Physics, Boston, 02115 MA, USA ${ }^{k}$

19) Northwestern University, Department of Physics, Evanston, $60208 \mathrm{IL}, \mathrm{USA}^{\mathrm{j}, \mathrm{k}}$

20) Rice University, Bonner Laboratory, Houston, 77251-1892 TX, USA ${ }^{\mathrm{j}}$

21) DAPNIA, CEN Saclay, 91191 Gif-sur-Yvette, France

22) University of Santiago, Department of Particle Physics, 15706 Santiago de Compostela, Spain ${ }^{\mathrm{n}}$

23) Tel Aviv University, School of Physics, 69978 Tel Aviv, Israel ${ }^{\circ}$

24) INFN Trieste and University of Trieste, Department of Physics, 34127 Trieste, Italy

25) Uppsala University, Department of Radiation Sciences, 75121 Uppsala, Sweden

26) University of Virginia, Department of Physics, Charlottesville, 22901 VA, USA ${ }^{\mathrm{k}}$

27) Warsaw University and Soltan Institute for Nuclear Studies, 00681 Warsaw, Poland ${ }^{p}$

28) Yale University, Department of Physics, New Haven, 06511 CT, USA ${ }^{\mathrm{j}}$

a) Now at CERN, 1211 Geneva 23, Switzerland

b) Now at University of Montreal, PQ, H3C 3J7, Montreal, Canada

c) Now at Max Planck Institute, Heidelberg, Germany

d) Permanent address: Miyazaki University, 88921 Miyazaki-Shi, Japan

e) Permanent address: PSI, 8093 Villigen, Switzerland

f) Permanent address: KEK, 305 Ibaraki-Ken, Japan

g) Permanent address: University of Buenos Aires, Physics Department, 1428 Buenos Aires, Argentina

h) Now at SSC Laboratory, Dallas, 75237 TX, USA

i) Supported by Bundesministerium für Forschung und Technologie

j) Supported by the Department of Energy

k) Supported by the National Science Foundation

l) Supported by Ishida Foundation, Mitsubishi Foundation and Monbusho International Science Research Program

m) Supported by the National Science Foundation of the Netherlands

n) Supported by Comision Interministerial de Ciencia y Tecnologia

o) Supported by the US-Israel Binational Science Foundation and The Israeli Academy of Sciences 
Measurements of deep inelastic scattering of polarized muons from polarized protons and deuterons determine the spin dependent structure functions of the nucleon which allow fundamental tests of quantum chromodynamics and of models of nucleon structure [1]. The precision of these experiments is strongly related to the polarization of the target nucleons. Therefore, the large enhancement of our deuteron target polarization which we discovered during the data-taking for deep inelastic muon scattering [2] had a significant impact on our experiment at CERN. The discovery was associated with a faulty regulator of the high voltage power supply of a microwave source. After controllable frequency modulation (FM) of the microwave tube was implemented, a gain by a factor of 1.7 in the maximum deuteron vector polarization and of 2.0 in the polarization growth rate were achieved. These increases have been of crucial importance because data-taking extends over many months and the statistical error is proportional to $1 / P N^{1 / 2}$, in which $P=\left\langle I_{z}\right\rangle / I$ is the target vector polarization and $\mathrm{N}$ is the number of scattered events. The magnitude of the enhancement has been reported earlier [3]. The purpose of this paper is to detail the full characteristics of this effect, to present new data on the electron paramagnetic resonance absorption (EPR) spectrum and to discuss briefly processes which may contribute to the FM phenomenon.

The polarized target [4, 5] consists of two cells each $40 \mathrm{~cm}$ long and $5 \mathrm{~cm}$ in diameter located in a large cylindrical multimode microwave cavity. The two target halves are polarized in opposite directions by dynamic nuclear polarization (DNP). The target material is glassy, perdeuterated 1-butanol, $\mathrm{C}_{4} \mathrm{D}_{9} \mathrm{OD}$ with $5 \%$ by weight of deuterium oxide, doped with the paramagnetic EDBA-Cr(V) complex [6] to a concentration of $7 \cdot 10^{19} \mathrm{~cm}^{-3}$ [7]. It is located in a magnetic field of $2.5 \mathrm{~T}$ with a uniformity of $10^{-4}$ over the volume and is cooled by a dilution refrigerator. The DNP is obtained by applying microwave power near the EPR frequency of the paramagnetic complex.

The deuteron vector polarization is measured with nuclear magnetic resonance (NMR) probes, each of which is part of a series tuned Q-meter circuit [8]. The material is sampled by five probes in each target cell. The polarization is determined from the integrated NMR signals, calibrated in thermal equilibrium at $1 \mathrm{~K}$. The relative accuracy of the measurement $\delta P / P$ is $5 \%$ [3].

The microwave power for DNP is produced by two extended interaction oscillators (EIO) with an emission bandwidth of about $0.1 \mathrm{MHz}$. The rate of polarization is optimized by controlling the microwave power and frequency. The frequency is controlled by the EIO cathode voltage with a sensitivity of about $0.4 \mathrm{MHz} / \mathrm{V}$ or by tuning the EIO cavity. The power is controlled by non-reflective attenuators.

For materials in which the solid effect 99, 10 dominates as a mechanism for DNP, it has been found that microwave FM can improve the rate of DNP. This appears to result from the fact that FM counteracts the effect of "hole burning" due to EPR absorption at a fixed frequency [11. In the glassy alcohol materials with $\mathrm{Cr}(\mathrm{V})$ complexes, where the dynamic nuclear cooling [12] is the dominant mechanism for DNP, hole burning is not expected. However a polarization enhancement of $10 \%$ to $20 \%$ was observed in a fluorinated alcohol leading to polarizations of $\approx 0.80$ for protons and ${ }^{19} \mathrm{~F}[13]$. References to enhancements of a few percent at polarizations around 0.70 or of about $15 \%$ for a material with only a few percent polarization can also be found in [13]. To our knowledge no studies have been reported for the effect of FM on deuteron polarization except in one

p) Supported by KBN

q) Now at Technische Universität, Dresden, D-01062 
case where FM was used to compensate for magnetic field inhomogeneity and improve the final polarization by $5 \%$ to $\approx 0.30$ [14].

The large enhancement of deuteron polarization in our target due to FM came therefore as a surprise. Figure 1 shows the typical time evolution of the deuteron polarization $P_{D}$ without and with FM. For this figure the cathode voltages were modulated at $1 \mathrm{kHz}$ with $\mathrm{a} \approx 50$ volt peak-to-peak amplitude leading to a FM amplitude $\Delta f \approx 20 \mathrm{MHz}$ for the $69 \mathrm{GHz}$ microwave source. The maximum deuteron vector polarizations under these conditions were 0.43 and -0.49 .

The EPR spectrum was measured in our target at a constant frequency by scanning the magnetic field. Such a spectrum, shown in Figure 2 without FM, was obtained using a $220 \Omega$ Speer composite carbon resistor as a bolometer, located in the dilute phase of the mixing chamber outside the target material [15]. The input power to the microwave cavity $\dot{Q}_{I N}$ is the sum of $\dot{Q}_{M A T}$, the power absorbed by the material in the EPR process, and $\dot{Q}_{N R}$ the non-resonant power absorbed into the cavity. The power absorbed by the bolometer $\dot{Q}_{S P}$ is a constant fraction $r$ of $\dot{Q}_{N R}$. It can be expressed as $\dot{Q}_{S P}=c\left(T_{S P}^{4}-T_{H E}^{4}\right)$ where $T_{S P}$ is the temperature of the bolometer, $T_{H E}$ is the temperature of the dilute phase and $c$ is a constant [16]. During the EPR measurement the input power $\dot{Q}_{I N}$ remains constant and we can neglect the variations of $T_{H E}^{4}$. Consequently the relation $\dot{Q}_{I N}=$ $\dot{Q}_{M A T}+\dot{Q}_{N R}=\dot{Q}_{M A T}+(c / r) \times\left(T_{S P}^{4}-T_{H E}^{4}\right)$ shows that $\dot{Q}_{M A T}$ is a linear function of $T_{S P}^{4}$. The broad absorption band seen in Figure 2 is due to the anisotropy of the $g$-factor of the EDBA-Cr(V) electron spin. The highest positive and negative polarizations without FM were obtained at frequencies $f_{0}^{+}=69.090 \mathrm{GHz}$ and $f_{0}^{-}=69.520 \mathrm{GHz}$, respectively.

The EPR spectra with better resolution at the edges of the absorption band are shown in Figure $3 \mathrm{a}$ and $3 \mathrm{~b}$, both with and without FM. The data points with FM were obtained using a modulation amplitude $\Delta f=4 \mathrm{MHz}$ to keep a good resolution in our spectra. In order to measure the small change in EPR aborption due to FM a novel technique of making consecutive measurements of the bolometer resistance with and without FM at each field step was employed. In Figures $3 \mathrm{c}$ and $3 \mathrm{~d}$ we display the difference $\Delta T_{S P}^{4}=\left(T_{S P}^{\text {off }}\right)^{4}-\left(T_{S P}^{\text {on }}\right)^{4}=\left(\dot{Q}_{M A T}^{\text {on }}-\dot{Q}_{M A T}^{\text {off }}\right) / c$. These data demonstrate that FM increases $\dot{Q}_{M A T}$ in the edges of the EPR spectrum. Note that the structures in Figure 3a and $3 \mathrm{~b}$ which extend down to $69.00 \mathrm{GHz}$ and up to $69.60 \mathrm{GHz}$ are almost entirely eliminated in the presence of FM even though the amplitude of FM is small compared to their width.

In Figure 4 we show the difference $\Delta T_{S P}^{4}$ as a function of the frequency of $\mathrm{FM}$ for different input power levels $\dot{Q}_{I N}$ with an FM amplitude $\Delta f=30 \mathrm{MHz}$ at $69.090 \mathrm{GHz}$ where $\Delta T_{S P}^{4}$ reaches a maximum. This difference grows with the modulation frequency up to a maximum value (indicated by the arrows) and then remains constant. The frequencies at which the additional EPR absorption reaches its maximum value increase roughly linearly with $\dot{Q}_{I N}$. A study of the polarization growth rate was performed at high negative $P_{D}$ values for a setting of $\dot{Q}_{I N}$ close to the one which was used for curve 2 of Fig.4. The rate increased with modulation frequency and reached a maximum value of $-0.8 \%$ per hour when modulating at $10 \mathrm{~Hz}$. At this $\dot{Q}_{I N}$ value, $\Delta T_{S P}^{4}$ reaches a maximum at this frequency which suggests strongly that the additional EPR absorption due to FM is what leads to the enhanced DNP.

In further measurements, we have established that the highest positive and negative polarizations with $\mathrm{FM}$ were obtained using $\Delta f \approx 30 \mathrm{MHz}$ at $f_{0}^{+}=69.070 \mathrm{GHz}$ and $f_{0}^{-}=69.540 \mathrm{GHz}$, respectively. The gain in maximum polarization due to $\mathrm{FM}$ is 1.7 and the increase in polarizing speed is about two. The homogeneity of the deuteron 
polarization throughout the target volume was investigated . Two radially superimposed coils measuring polarization at radii of $1.5 \mathrm{~cm}$ and $0.5 \mathrm{~cm}$ showed a deuteron polarization ratio from the small to the large coil of 1.20 before and 1.06 after applying FM [3]. A study of the deuteron NMR line asymmetry [3] provided us with an upper limit $\Delta P_{D}$ for the spatial variation of $P_{D}$. Typical values for $\Delta P_{D}$ were 0.30 without FM and 0.15 with FM which confirmed that FM improves the uniformity of polarization.

The large enhancement has been confirmed recently in our new $2.5 l$ target [17 where maximum deuteron polarizations of 0.46 and -0.60 were observed using FM. In spite of the improved magnetic field uniformity of $3.10^{-5}$ of the new target, the optimum $\Delta f / f$ for $\mathrm{FM}$ was about $0.5 \cdot 10^{-3}$ as in the previous target with $10^{-4}$ field uniformity. Also we find that $\Delta f / f$ is large compared to the target magnetic field inhomogeneity in both targets. We conclude that the mechanism by which FM improves polarization has little to do with the field nonuniformity. For protons FM increased the polarization, typically from 0.75 to 0.85 , and to maximum values as high as 0.95 18.

The existing theory [19, 20, 21 provides a qualitative understanding of the DNP for our target material; however, the large polarization enhancement due to microwave FM may require additional mechanisms. An example is the cross-relaxation within the system of electron spins which has been assumed to be fast. It has been suggested [22] that a slow cross-relaxation may lead to a lack of thermal equilibrium among electron spins and hence to unequal spin temperatures for different nuclei which results in lower nuclear polarization. FM may counteract this effect by increasing the number of electron spins which are saturated.

A possibly related effect is the local depletion of the electron spin packets which has been observed for materials whose EPR lines are broadened by hyperfine interactions when irradiated at fixed frequency. With FM, this local depletion can be avoided and a migration of spin packets occurs towards the wings of the EPR band [23]. This may result in a stronger EPR absorption in the wings.

Since the aim of our experiment was to measure spin-dependent asymmetries in polarized deep inelastic scattering, we did not attempt a more detailed study of the effect of FM on the target polarization. Our observations of the EPR absorption were used as a guide to optimize the parameters of the FM.

In conclusion, we discovered a large increase in deuteron polarization due to frequency modulation which is of great value for our high energy physics experiment. We found that an amplitude of $\mathrm{FM}$ of $\approx 30 \mathrm{MHz}$ and a frequency of $1 \mathrm{kHz}$ improved the deuteron polarization growth rate by a factor of 2 and resulted in deuteron polarizations as high as 0.60 with improved spatial uniformity over the target volume. Relations of this large FM effect to features of the EPR absorption mechanism were found and may provide useful information to a deeper understanding of dynamic nuclear polarization. 


\section{References}

[1] V.W. Hughes and J. Kuti, Ann. Rev. Nucl. Part. Sci. 33 (1983) 611.

[2] Spin Muon Collaboration, B. Adeva et al., Phys. Lett. B 302 (1993) 533.

[3] Spin Muon Collaboration, B. Adeva et al., Nucl. Inst. Meth. A349 (1994) 334.

[4] T. Niinikoski Nucl. Inst. Meth. 192 (1982) 151.

[5] S. C. Brown et al., in Proceedings of the 4th Int. Workshop on Polarized Target Material and Techniques, Bad Honnef (Germany), edited by W. Meyer (3-6 Sept. 1984) p. 102.

[6] M. Krumpolc, D. Hill and H.B. Stuhrmann, in Proceedings of the 9th Int. Symposium on High Energy Spin Physics, volume 2 Bonn (Germany), edited by W. Meyer, E. Steffens and W. Thiel (6-15 Sept. 1990) p. 340.

[7] S.Bültmann. 7th Workshop On Polarized Target Materials and Techniques, Bad Honnef (Germany), 3-6 June 1994, Nucl. Inst. Meth. A356 (1995) 102.

[8] G.R. Court et al., Nucl. Inst. Meth. A324 (1993) 443.

[9] A. Abragam and M. Goldman, Rep. Prog. Phys. 41 (1978) 395.

[10] T. J. Schmugge and C. D. Jeffries, Phys. Rev. 138 (1965) A1785.

[11] H.W. van Kesteren, W.Th. Wenckebach and J. Schmidt, Chem. Phys. Lett. 121 (1985) 440.

[12] A. Abragam and M. Borghini, Dynamic Polarization of Nuclear Targets, in Progress in Low-Temperature Physics Vol 4, Ed. C. J. Gorter (North Holland Publ. Co., 1964) p. 384 .

[13] D. Hill et al., Nucl. Inst. Meth. A277 (1989) 319. and references therein.

[14] S. Ishimoto et. al., Jap. J. Appl. Phys. 28 (1989) 1963.

[15] T.O. Niinikoski and J.M. Rieubland, Phys. Lett. 72A (1979) 141.

[16] O.V. Lounasmaa, Experimental Principles and Methods Below 1K, (Academic Press, London 1974) p. 265.

[17] J. Kyynäräinen. 7th Workshop On Polarized Target Materials and Techniques, Bad Honnef, Germany, 3-6 June 1994, Nucl. Inst. Meth. A356 (1995) 102.

[18] Spin Muon Collaboration, D. Adams et al., Phys. Lett. B 329 (1994) 395.

[19] B.N. Provotorov, Soviet Phys. JETP 14 (1962) 1126.

[20] B.N. Provotorov, Soviet Phys. JETP 15 (1962) 611.

[21] M.A. Kozhushner and B.N. Provotorov Soviet Phys. Solid State 6 (1964) 1152.

[22] T.O. Niinikoski, 10th International Symposium on High Energy Spin Physics, Nagoya, Japan, 9-14 November (1992).

[23] M. Goldman et al., J. Physique 30 (1969) 427. 


\section{Figure Caption}

\section{Figure 1}

Deuteron polarization as a function of time without FM (dark circles) and with FM (open circles). Positive and negative polarizations are shown.

\section{Figure 2}

Electron paramagnetic resonance absorption band for the glassy perdeuterated butanol of the SMC polarized target (the dotted line guides the eye). The temperature $T_{S P}$ is derived from the value of a Speer carbon composite resistor located near the material. The measurements were performed at a constant frequency $f_{0}=69.520 \mathrm{GHz}$ by step-

ping the magnetic field. The field values $H$ are converted to the equivalent frequencies $f=f_{0} H / H_{0}$ at $H_{0}=2.5 \mathrm{~T}$.

\section{Figure 3}

Enhancement in the wings of the EPR absorption spectrum observed when the microwave frequency was modulated with an amplitude of $4 \mathrm{MHz}$ at $1 \mathrm{kHz}$ frequency. Figures a and b show the EPR spectra obtained without (dark circles) and with FM (open circles) for the domain of frequency leading to positive (a) and negative (b) polarizations. Figures $\mathrm{c}$ and $\mathrm{d}$ show the differential effect.

\section{Figure 4}

Enhancement of the EPR absorption as a function of the FM frequency for different values of the input microwave power $\dot{Q}_{I N}$. The arrows show the frequencies at which the maximum enhancement is reached. The four curves labelled 1, 2, 3 and 4 were obtained at levels of input power $\dot{Q}_{I N}$ increased successively by a factor 4 . 
FIGURE 1

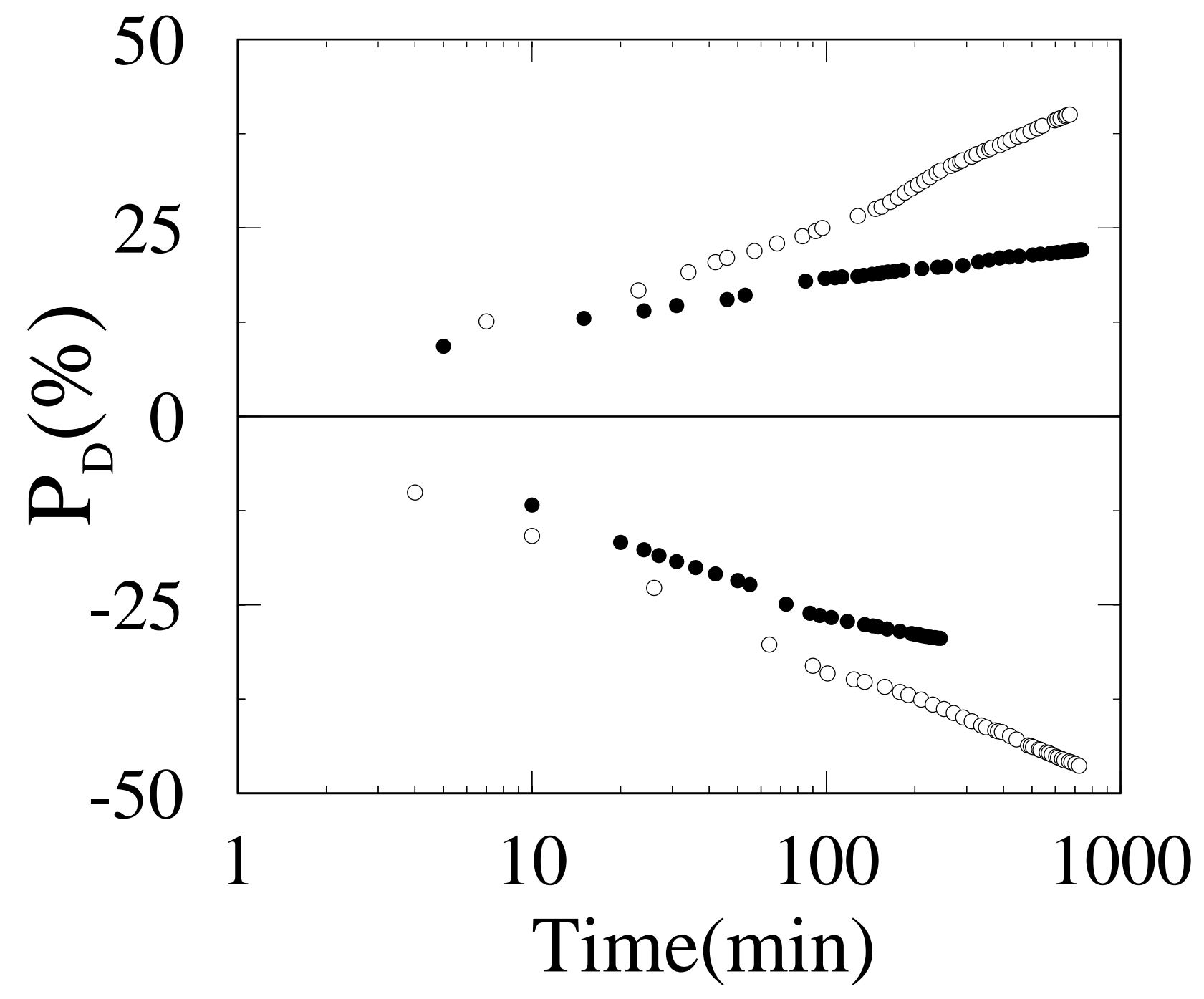


FIGURE 2

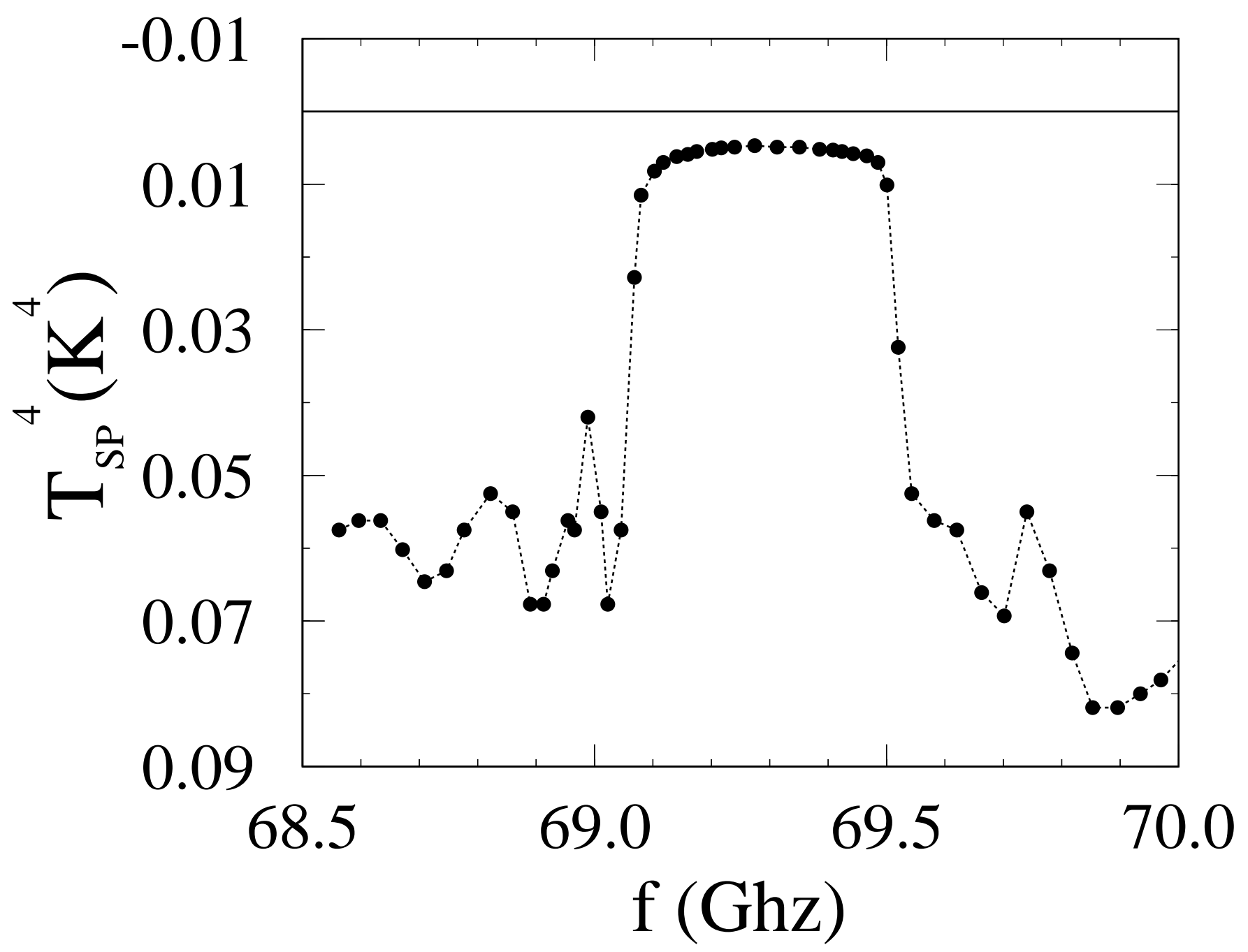


FIGURE 3

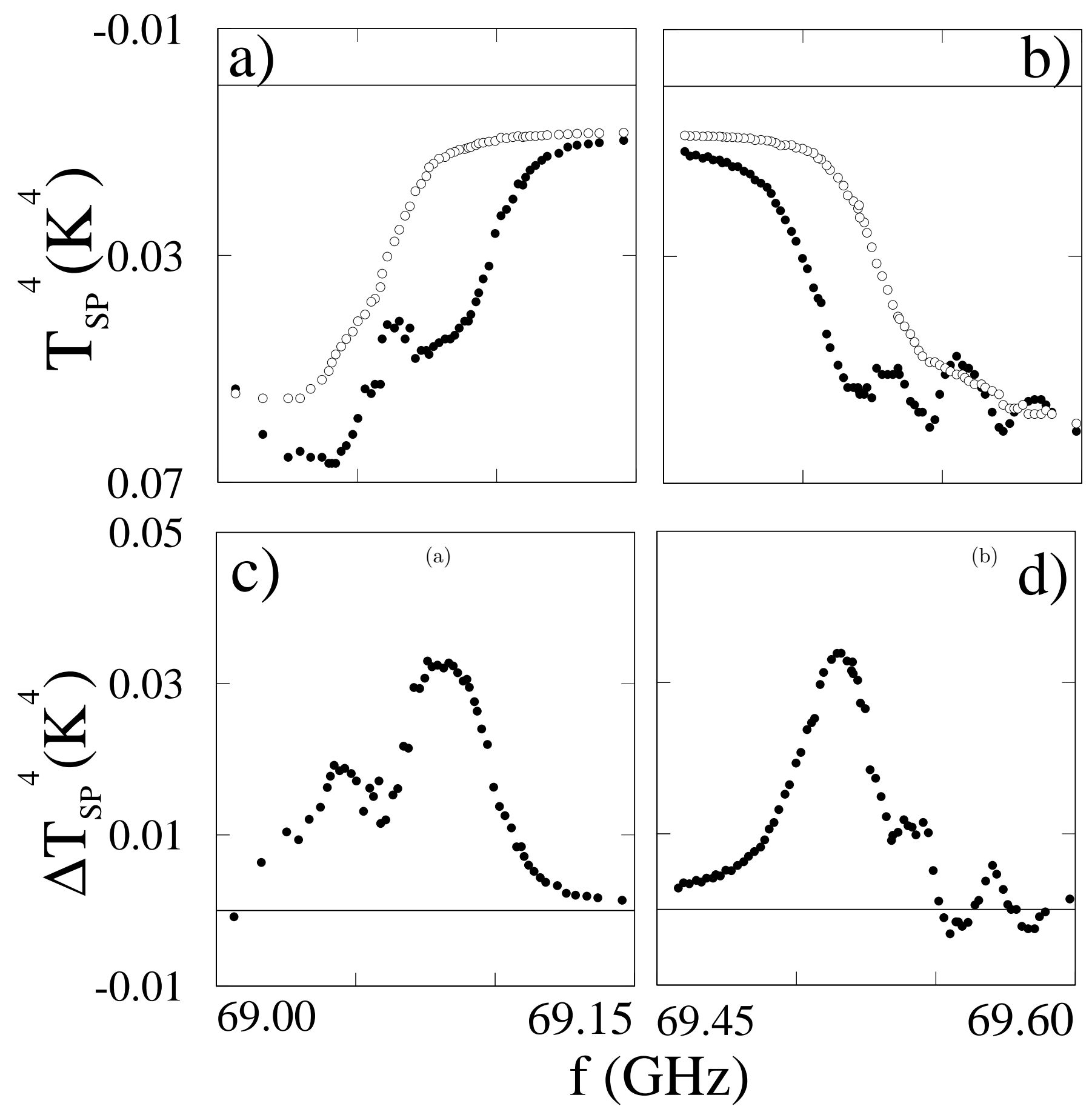

(c)

(d) 
FIGURE 4

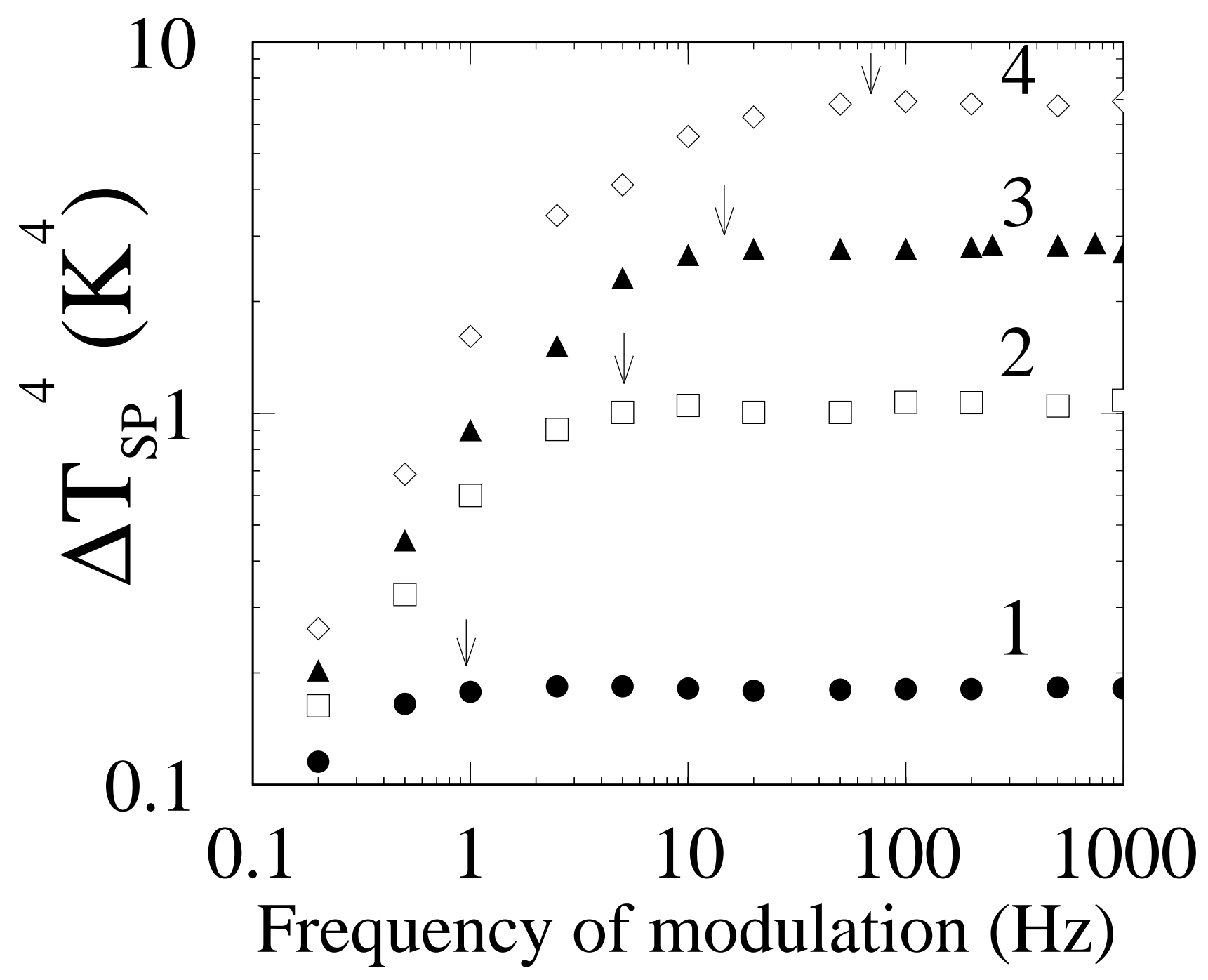

